\title{
Intermediate frequency electrotherapy stimulation to the medial femoris muscle for functional recovery of knee joint after anterior cruciate ligament reconstruction
}

\author{
Dejun Song ${ }^{1}$, Yubao $M a^{2}$, Lihua Zhang ${ }^{3}$, Quansheng $M a^{4}$
}

\begin{abstract}
Objectives: To compare the effect of medial femoral muscle stimulation with medium frequency electrotherapy and conventional rehabilitation therapy on knee function recovery after anterior cruciate ligament $(\mathrm{ACL})$ reconstruction.

Methods: Medical records of 50 patients with ACL reconstruction, treated in our hospital between July 2019 and December 2020, were retrospectively analyzed. Patients were divided into control group and study group ( $n=25,18$ males and 7 females in each group), based on the rehabilitation method used. The control group included patients that received conventional rehabilitation therapy, active quadriceps femoris exercise, traction, and acupuncture. The study group included patients that received medium frequency electrotherapy to stimulate the medial femoris muscle in addition to conventional rehabilitation therapy. The limb circumference recorded before and after the treatment was compared between the two group. The Lysholm scores of the two groups were compared to assess knee function, knee range of motion, and knee motor comfort assessed by visual analogue scale (VAS).

Results: We found similar thigh circumferences, Lysholm scores, knee motion ranges and VAS scores between the patients in both groups before the treatment $(P>0.05)$. After the treatment, the thigh circumferences and motion ranges were larger, the Lysholm scores higher, and the VAS scores lower in patients of the study group than those in patients of the control group (all Ps <0.05).

Conclusion: Intermediate frequency electrotherapy to stimulate the medial femoris muscle can improve knee function and motion range and reduce the patient's pain after ACL reconstruction.
\end{abstract}

KEYWORDS: Anterior cruciate ligament reconstruction, Electrotherapy, Quadriceps muscle, Knee function.

How to cite this:

doi: https://doi.org/10.12669/pjms.38.3.5298

Song $D$, Ma $Y$, Zhang $L$, Ma Q. Intermediate frequency electrotherapy stimulation to the medial femoris muscle for functional recovery of knee joint after anterior cruciate ligament reconstruction. Pak J Med Sci. 2022;38(3):652-656. doi: https://doi.org/10.12669/pjms.38.3.5298

This is an Open Access article distributed under the terms of the Creative Commons Attribution License (http://creativecommons.org/licenses/by/3.0), which permits unrestricted use, distribution, and reproduction in any medium, provided the original work is properly cited.

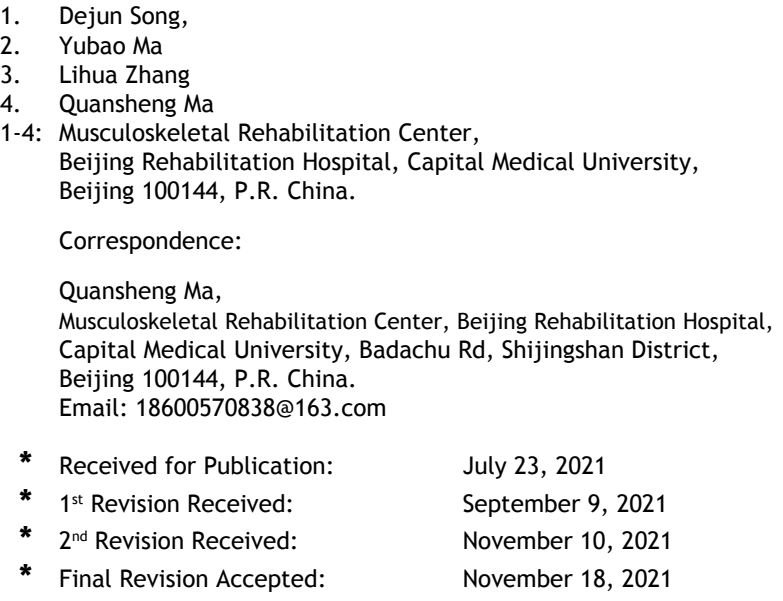

\section{INTRODUCTION}

The anterior cruciate ligament (ACL) of the knee is frequently injured during sports and military training activities, and this can lead to cartilage and meniscus injury and even to osteoarthritis. ${ }^{1,2}$ ACL reconstruction is a common treatment, but it diminishes the quadriceps femoris function causing lower limb muscle atrophy and functional degradation due to the routine leg immobilization brace use required after lower limb surgery. Therefore, rehabilitation exercises to promote the recovery of knee joint function are needed after reconstruction. ${ }^{3,4}$ 
An imbalance of muscle force between the medial oblique and lateral femoris muscles can lead to and can result in lateral patella tracking and pain during knee flexion-extension.,6 Transcutaneous electrical muscle stimulation (TEMS) has been used to rehabilitate the lower extremity function after ACL repair/reconstruction. ${ }^{7}$ Medium frequency electrical stimulation of the medial femoral muscle should be useful to improve knee function recovery after ACL reconstruction, but no studies have evaluated this approach. Therefore, we conducted this retrospective study to assess the effect of medium frequency electrical stimulation of the medial femoral muscle on knee function recovery after ACL reconstruction.

\section{METHODS}

Medical records of the patients with ACL reconstruction, treated in our hospital between July 2019 and December 2020, were retrospectively analyzed, a total of 50 patients met the inclusion criteria. Patients were divided into control group and study group $(n=25,18$ males and 7 females in each group), based on the therapeutic method used. Inclusion criteria: All participants had had a simple cruciate ligament rupture without meniscus, posterior cruciate ligament, or collateral ligament injury and they had undergone a single ACL reconstruction; their age ranged from 18 to 43 years; they all lacked a previous operation history. Exclusion criteria: We excluded patients with joint adhesions and vascular nerve injury; patients with heart disease, liver and kidney diseases; and patients with audiovisual and language impairments. According to the above criteria, records of 50 patients with ACL reconstruction in our hospital from July 2019 to December 2020 were selected for the study. The ethics committee of our hospital approved the study (Approval number: 21009, Date: 2021 June 20).

Therapeutic method: The control group included patients that were treated with conventional rehabilitation therapy sessions including active quadriceps femoris exercise, muscle traction, and acupuncture (30 minutes twice a day, 5 days a week, for 8 weeks).

The study group included patients that received the routine treatment in combination with the medium frequency pulse electrotherapy. After routine treatment, when the patient's vital signs were stable and the symptoms of nerve defect were not further aggravated, patients were treated with medium frequency pulse electrotherapy. BA2008-
III microcomputer bionic therapeutic instrument (Beijing BENAO New Technology Co., Ltd) was used to stimulate the medial femoral muscle. The treatment current of medium frequency electrical stimulation was the medium frequency current modulated by low frequency; The frequency range is set as the intermediate frequency (2000 $\sim 4000 \mathrm{~Hz}) .{ }^{8}$ The two output ends are respectively connected with rectangular electrode pieces. The muscle stimulation points are selected at the beginning of medial femoral muscle on the medial patella and $10 \mathrm{~cm}$ above the patella, which do not exceed the midline of the thigh. The conversion time between stimulation and rest was set to 15 $\mathrm{s}$; Take the patient's tolerable tingling, tremor, twitch and muscle contraction as the degree, which shall not exceed $70 \%$ of the maximum tolerable intensity with each stimulation lasting between 10 and 20 minutes (4 courses of daily stimulations for 10 days).

Observation Variables: Medical records of all the patients were analyzed for the following parameters:

1. The thigh circumference $10 \mathrm{~cm}$ above the upper edge of the patella before and after the treatment.

2. Lysholm scores to evaluate the knee joint function ${ }^{9}$ before and after treatment (the highest score being 100 for the best function).

3. Knee ranges of motion measurements before and after treatment. The larger the range of motion, the better the knee function.

4. A visual analogue scale (VAS) with 10 consecutive points (0 to 10 points) at equal intervals to allow patients to indicate their perceived knee joint comfort before and after treatment. The lower the score, the less comfort.

Statistical Analysis: Data was analyzed using the SPSS 19.0 statistical software. The measurement data expressed in $(\bar{x} \pm s)$. Two independent sample t-test is used for the comparison between the two groups, and paired t-test is used for the comparison before and after treatment in a single group. When $p<0.05$, it was considered as statistically significant.

\section{RESULTS}

Patients in the control group had a mean age of 32.24 years \pm 6.53 , a mean body weight of 77.44 $\mathrm{kg} \pm 1.87$, a mean height of $175.76 \mathrm{~cm} \pm 2.85$. The patients in the study group had a mean age of 32.52 years \pm 5.8 , a mean body weight of $77.48 \mathrm{~kg}$ \pm 1.71 , a mean height of $175.24 \mathrm{~cm} \pm$. We found no significant differences in the basic data 
Table-I: Comparison of thigh circumference changes in the affected

limbs between two groups before and after treatment $(\bar{x} \pm \mathrm{s}, \mathrm{cm})$.

\begin{tabular}{lccccc}
\hline Group & $n$ & Before treatment & After treatment & $t$ & $P$ \\
\hline Control group & 25 & $41.28 \pm 0.88$ & $42.30 \pm 0.89$ & 4.075 & $<0.001$ \\
Research Group & 25 & $41.30 \pm 0.73$ & $44.57 \pm 0.77$ & 15.409 & $<0.001$ \\
$t$ & & 0.087 & 9.644 & & \\
$P$ & & 0.931 & $<0.001$ & \\
\hline
\end{tabular}

$\mathrm{t}$ and $\mathrm{P}$-values represents the results after independent sample $\mathrm{t}$-test and paired sample $\mathrm{t}$-test, respectively.

Table-II: Comparison of Lysholm scores between the groups before and after treatment $(\bar{x} \pm s)$.

\begin{tabular}{lccccc}
\hline Group & $n$ & Before treatment & After treatment & $t$ & $P$ \\
\hline Control group & 25 & $51.27 \pm 5.74$ & $61.55 \pm 5.36$ & 6.545 & $<0.001$ \\
Research Group & 25 & $51.32 \pm 6.04$ & $67.86 \pm 6.28$ & 9.491 & $<0.001$ \\
$t$ & & 0.030 & 3.821 & & \\
$P$ & & 0.976 & $<0.001$ & & \\
\hline
\end{tabular}

$\mathrm{t}$ and $\mathrm{P}$-values represents the results after independent sample $\mathrm{t}$-test and paired sample $\mathrm{t}$-test, respectively.

between the two groups $(\mathrm{P}>0.05)$. As shown in Table-I, we found similar circumferences before treatment $(\mathrm{P}>0.05)$. After the treatment, the thigh circumferences of the affected limb in the study group were significantly larger than those in the control group $(\mathrm{P}<0.05)$. We found similar Lysholm scores between the control group and the study group before treatment $(\mathrm{P}>0.05)$. After treatment, the Lysholm scores in the study group were significantly higher than those in the control group (P< 0.05), Table-II.

Motion ranges was also similar before treatment $(P>0.05)$. After treatment, the motion ranges in the study group were significantly higher than those in the control group $(\mathrm{P}<0.05)$, Table-III VAS scores was also similar before treatment $(\mathrm{P}>0.05)$, Table-IV. After treatment, the VAS scores in the study group were significantly lower than those in the control group $(\mathrm{P}<0.05)$.

\section{DISCUSSION}

The stability of the knee during joint flexion depends on the bone placement, on the restriction of the anterior and posterior cruciate ligaments, and on the balance of the strengths of the quadriceps femoris muscles. After an ACL reconstruction, the biomechanical structure of the lower limb is significantly altered, and the afferent and efferent action potentials of the nerves in the affected limb are significantly reduced, inhibiting the signal transduction and feedback pathway of the knee joint nervous system. ${ }^{10,11}$ Moreover, damage to the muscles around the knee can lead to the patients ineffectively maintaining their body balance and the knee injury aggravated. ${ }^{12}$ Correct postoperative nursing and rehabilitation interventions can effectively prevent postoperative complications

Table-III: Comparison of knee motion ranges before and after treatment between the groups $(\bar{x} \pm s)$.

\begin{tabular}{lccccc}
\hline Group & $n$ & Before treatment & After treatment & $t$ & $P$ \\
\hline Control group & 25 & $44.39 \pm 4.53$ & $57.34 \pm 5.73$ & 8.865 & $<0.001$ \\
Research Group & 25 & $44.42 \pm 4.62$ & $66.33 \pm 6.01$ & 14.451 & $<0.001$ \\
$\mathrm{t}$ & & 0.023 & 5.413 & & \\
$\mathrm{P}$ & & 0.982 & $<0.001$ & \\
\hline
\end{tabular}

$\mathrm{t}$ and $\mathrm{P}$-values represents the results after independent sample $\mathrm{t}$-test and paired sample $\mathrm{t}$-test, respectively. 
Dejun Song et al.

Table-IV: Comparison of VAS scores between the groups before and after treatment $(\bar{x} \pm \mathrm{s})$.

\begin{tabular}{lccccc}
\hline Group & $n$ & Before treatment & After treatment & $t$ & $P$ \\
\hline Control group & 25 & $7.73 \pm 1.75$ & $5.39 \pm 1.08$ & 5.689 & $<0.001$ \\
Research Group & 25 & $7.89 \pm 1.68$ & $4.03 \pm 1.02$ & 9.820 & $<0.001$ \\
$\mathrm{t}$ & & 0.330 & 4.577 & & \\
$\mathrm{P}$ & & 0.743 & $<0.001$ & & \\
\hline
\end{tabular}

t and P-values represents the results after independent sample t-test and paired sample t-test, respectively.

and promote knee function recovery. ${ }^{13}$ Thus, a rehabilitation plan based on sound scientific and medical technologies based on biodynamics and anatomy can help the healing process and function recovery after knee joint injury and reconstruction. ${ }^{14}$

The biomechanical changes after ACL reconstruction reduce the nerve muscle control and cause quadriceps heads' asymmetry, which can easily cause secondary knee injuries. ${ }^{15}$ During physiotherapy with electrical stimulation, an electrical current causes muscle contraction, and the current frequency can be modulated to produce low and medium waveforms. The exciting effect on the muscle can enhance the local metabolism, and adaptation is avoided by the application of multiple waves. ${ }^{16,17}$ Neuromuscular electrical stimulation on the basis of functional exercise can increase muscle strength and improve limb asymmetry after ACL reconstruction. However, the optimal electrical stimulation frequency varies with the treatment target. ${ }^{18}$ Studies have shown that $2-30 \mathrm{~Hz}$ low frequency electrical stimulation may improve the hypoxia tolerance, treadmill fatigue, and swimming exhaustion times in mice; while 10$100 \mathrm{~Hz}$ low-frequency electrical stimulation can increase the fibrin content in the gastrocnemius muscle and promote the synthesis of skeletal muscle. ${ }^{19,20}$ In our study, a combination of the low-frequency modulated medium frequency electrical stimulation (medium frequency, 2000$4000 \mathrm{~Hz}$ and low frequency, 1-167 Hz) was used. This frequency does not stimulate the skin sensory nerve endings and does not cause pain, which makes it ideal to ensure patient tolerance. ${ }^{21}$ Electrical muscle stimulation can improve tension in atrophic muscles and the stability of bones and joints. Under the action of a pulse current, the medial femoral muscle will contract intermittently, promoting the circulation of joint fluid and articular cartilage, and promoting the knee joint recovery. ${ }^{22}$ The technology is well designed and simple and causes no injury or pain, leading to patient compliance. ${ }^{23}$ Our results showed that after treatment, the thigh circumferences, Lysholm scores and knee motion ranges were higher and the pain reduced to a higher degree in the patients that received medium frequency electrotherapy in addition to conventional rehabilitation therapy than in the patients who received conventional rehabilitation therapy alone. Our findings suggest that medial femoris muscle stimulation by medium frequency electrotherapy improves the knee function and motion range of patients after ACL reconstruction.

Limitations of the study: The main limitations of this study are the small sample size (only 50 patients) and its retrospective nature. Larger, prospective, and retrospective studies, as well as clinical trials are needed to further evaluate the therapeutic effect of medium frequency electrical stimulation in patients after ACL reconstruction.

\section{CONCLUSION}

Medium frequency electrical stimulation of the medial femoral muscle can improve knee function and motion range and reduce the pain of patients after ACL reconstruction.

\section{REFERENCES}

1. Cheng YTY, Wong TKS, Tsang WWN, Schooling CM, Fong SSM, Fong DYT, et al. Neuromuscular training for children with developmental coordination disorder: A randomized controlled trial. Medicine (Baltimore). 2019;98(45):e17946. doi: 10.1097/MD.0000000000017946

2. Ham S, Kim S, Choi H, Lee Y, Lee H. Greater Muscle Stiffness during Contraction at Menstruation as Measured by Shear-Wave Elastography. Tohoku J Exp Med. 2020;250(4):207-213. doi: 10.1620/tjem.250.207

3. Chen LX, Wang HH. Comparison between single- and double-bundle anterior cruciate ligament reconstructions for knee with grade 2 medial collateral ligament injury. Med (Baltimore). 2021;100(11):e24846. doi: 10.1097/ MD.0000000000024846 
4. Hurley ET, Fried JW, Kingery MT, Strauss EJ, Alaia MJ. Antero-lateral ligament reconstruction improves knee stability alongside anterior cruciate ligament reconstruction. Knee Surg Sports Traumatol Arthrosc Off J ESSKA. 2021;29(3):764-771. doi: 10.1007/s00167-02006002-8

5. Yoon KH, Lee HW, Park SY, Yeak RDK, Kim JS, Park JY. Meniscal Allograft Transplantation After Anterior Cruciate Ligament Reconstruction Can Improve Knee Stability: A Comparison of Medial and Lateral Procedures. Am J Sports Med. 2020;48(10):2370-2375. doi: $10.1177 / 0363546520938771$

6. Hamrin Senorski E, Svantesson E, Baldari A, Ayeni OR, Engebretsen L, Franceschi F, et al. Factors that affect patient reported outcome after anterior cruciate ligament reconstruction-a systematic review of the Scandinavian knee ligament registers. Br J Sports Med. 2019;53(7):410417. doi: $10.1136 /$ bjsports-2017-098191

7. Kain CC, McCarthy JA, Arms S, Pope MH, Steadman JR, Manske PR, et al. An in vivo analysis of the effect of transcutaneous electrical stimulation of the quadriceps and hamstrings on anterior cruciate ligament deformation. Am J Sports Med. 1988;16(2):147-152. doi: $10.1177 / 036354658801600210$

8. Selkowitz DM, Rossman EG, Fitzpatrick S. Effect of burstmodulated alternating current carrier frequency on current amplitude required to produce maximally tolerated electrically stimulated quadriceps femoris knee extension torque. Am J Phys Med Rehabil. 2009;88(12):973-978. doi: 10.1097/PHM.0b013e3181c1eda5

9. Wang J, Wright KT, Perry J, Tins B, Hopkins T, Hulme $\mathrm{C}$, et al. Combined Autologous Chondrocyte and Bone Marrow Mesenchymal Stromal Cell Implantation in the Knee: An 8-year Follow Up of Two First-InMan Cases. Cell Transplant. 2019;28(7):924-931. doi: $10.1177 / 0963689719845328$

10. Murray MM, Fleming BC, Badger GJ, Team BT, Freiberger C, Henderson R, et al. Bridge-Enhanced Anterior Cruciate Ligament Repair Is Not Inferior to Autograft Anterior Cruciate Ligament Reconstruction at 2 Years: Results of a Prospective Randomized Clinical Trial. Am J Sports Med. 2020;48(6):1305-1315. doi: 10.1177/0363546520913532

11. Mouarbes D, Menetrey J, Marot V, Courtot L, Berard E, Cavaignac E. Anterior Cruciate Ligament Reconstruction: A Systematic Review and Meta-analysis of Outcomes for Quadriceps Tendon Autograft Versus Bone-Patellar Tendon-Bone and Hamstring-Tendon Autografts. Am J Sports Med. 2019;47(14):3531-3540. doi: 10.1177/0363546518825340

12. Amin I, Perveen W, Ali MA, Ilyas U. Heterotrophic calcification of medial collateral ligament of knee joint: A case report. J Pak Med Assoc. 2021;71(2(B)):756-758. doi: 10.47391/JPMA.1082

13. Ghosh K, Salmon LJ, Heath E, Pinczewski LA, Roe JP. Transphyseal anterior cruciate ligament reconstruction using living parental donor hamstring graft: Excellent clinical results at 2 years in a cohort of 100 patients. Knee Surg Sports Traumatol Arthrosc Off J ESSKA. 2020;28(8):2511-2518. doi: 10.1007/s00167-019-05842-3

14. Do Cho S, Youm YS, Park SJ, Cho HI. Pigmented villonodular synovitis after anterior cruciate ligament reconstruction using active bioprosthetic composite artificial ligament. Rheumatol Int. 2020;40(4):657-661. doi:10.1007/s00296-019-04491-6
15. Lind M, Strauss MJ, Nielsen T, Engebretsen L. Quadriceps tendon autograft for anterior cruciate ligament reconstruction is associated with high revision rates: results from the Danish Knee Ligament Registry. Knee Surg Sports Traumatol Arthrosc Off J ESSKA. 2020;28(7):21632169. doi: 10.1007/s00167-019-05751-5

16. Kang HW, Kim HJ, Kim WY, Min WK, Min TJ, Lee YS, et al. Effects of cranial electrotherapy stimulation on preoperative anxiety and blood pressure during anesthetic induction in patients with essential hypertension. J Int Med Res. 2020;48(8):300060520939370. doi: $10.1177 / 0300060520939370$

17. Ferrari G, Colucci A, Barbariga M, Ruggeri A, Rama P. High Frequency Electrotherapy for the Treatment of Meibomian Gland Dysfunction. Cornea. 2019;38(11):14241429. doi: 10.1097/ICO.0000000000002063

18. Fouré A, Ogier AC, Guye M, Gondin J, Bendahan D. Muscle alterations induced by electrostimulation are lower at short quadriceps femoris length. Eur J Appl Physiol. 2020;120(2):325-335. doi: 10.1007/s00421-019-04277-5

19. Geney-Castro DE, Vanegas-Munoz J, Plata-Contreras J, Salinas-Duran F. Medial femoral cutaneous nerve conduction study with distal recording: A novel technique. Muscle Nerve. 2020;61(3):383-386. doi: 10.1002/mus.26788

20. Frazer LL, Santschi EM, Fischer KJ. Stimulation of subchondral bone cyst healing by placement of a transcondylar screw in the equine medial femoral condyle. Vet Surg VS. 2019;48(7):1194-1203. doi: 10.1111/vsu.13247

21. Li K, Li L, Liang L. Systematic evaluation and Meta analysis of percutaneous electrical stimulation for improving pain and function after total knee arthroplasty. J Hainan Med Univ 2019;24:1872-1877.

22. Zhang P, Liu P, Li H. The effect of percutaneous electrical acupoint stimulation on the effect of patient-controlled intravenous analgesia in elderly patients after total knee arthroplasty. J Clin Anesthesiol. 2019;3(35):243-246.

23. Li C, Sui H, Yu L. Study on gait regulation of stroke patients with functional electrical stimulation based on walking pattern. Chinese J Rehab Med. 2019;5(34):70-73.

\section{Authors' Contributions:}

DS and QM: Conceived and designed the study. YM \& LZ: Collected the data and performed the analysis.

DS and QM: Prepared the manuscript and is responsible for the integrity of the study.

All authors have read and approved the final manuscript. 